\title{
Recomendaciones ESRA/ASRA en anestésicos locales y adyuvantes empleados en la población pediátrica
}

Artículo original: The European Society of Regional Anaesthesia and Pain Therapy/AmericanSociety of Regional Anesthsia and Pain Medicine Recommendations on Local Anesthetics and Adjuvants Dosage in Pediatric Regional Anesthesia. Suresh S, Ecoffey C, Bosenberg A, Lonnqvist PA, de Oliveira GS Jr, de Leon Casasola O, et al. (PubMed)

Salmerón Martín M, Torres Mosquera P.

H.C.U. Virgen de la Arrixaca, Murcia.

\section{Resumen}

Dada la preocupación creciente respecto al efecto de los anestésicos empleados en anestesia general en el neurodesarrollo de la población pediátrica; toma mayor importancia la Anestesia Locorregional en dicha población. Pero ¿qué sabemos sobre la dosificación en esta población de los anestésicos locales? Debido a dicha incertidumbre y a lo poco publicado al respecto la ESRA (European Society of Regional Anesthesia) y la ASRA (American Society of Regional Anesthesia) han decido reunirse para establecer una serie de recomendaciones sobre la dosificación de anestésicos locales y adyuvantes en anestesia locorregional en la población pediátrica. Dicho documento lo hemos resumido, de forma esquemática y con ayuda de tablas, con el fin de facilitar su comprensión y tenerlo a la disposición de todo aquel interesado en este campo.

\section{Introducción}

Dada la preocupación creciente respecto al efecto de los anestésicos empleados en anestesia general en el neurodesarrollo de la población pediátrica; toma mayor importancia la Anestesia Locorregional en dicha población. Pero ¿qué sabemos sobre la dosificación en esta población de los anestésicos locales? Debido a dicha incertidumbre y a lo poco publicado al respecto la ESRA (European Society of Regional Anesthesia) y la ASRA (American Society of Regional Anesthesia) han decido reunirse para establecer una serie de recomendaciones sobre la dosificación de anestésicos locales y adyuvantes en anestesia locorregional en la población pediátrica. Dicho documento lo hemos resumido, de forma esquemática y con ayuda de tablas, con el fin de facilitar su comprensión y tenerlo a la disposición de todo aquel interesado en este campo.

La revista "Regional Anesthesia and Pain Medicine" F.I.: 3'464 nos trae un artículo en febrero del 2018 sobre el empleo de anestesia regional en la población pediátrica. Dado que el tema sobre neurotoxicidad infantil por anestesia general es una preocupación incipiente; la anestesia locorregional se convierte en una alternativa en esta población en determinadas cirugías.

Existen varios estudios que tratan sobre la neurotoxicidad de los fármacos que más comúnmente empleamos en nuestro día a día: propofol, sevorane, cisatracurio... Dichos artículos realizados en animales aún no se han podido demostrar objetivamente en humanos, pero ante el temor a este evento la FDA recomendó que las cirugías no urgentes que requieren 
anestesia se deben evitar en menores de 3 años. Sin embargo, la mayoría de las cirugías en niños jóvenes son urgentes por naturaleza.

El empleo de la anestesia locorregional siempre que sea posible como alternativa a la anestesia general es muy importante, sobre todo en la población infantil por lo dicho previamente. Las dosis de anestésicos locales no están bien determinadas en la población pediátrica; por ello, y para esclarecer este problema, se ha formado un comité por parte de la Sociedad Europea de Anestesia Regional y Terapia del dolor (ESRA) y la Sociedad Americana de Anestesia Regional y Medicina del dolor (ASRA) para establecer las dosis de anestésicos locales y adyuvantes que han de ser empleados en anestesia regional de la población pediátrica.

No solo han recurrido a recomendaciones basadas en la evidencia mediante la búsqueda sistemática bibliográfica; si no que aquellos casos de los que no se dispone de literatura se ha recurrido a comités de expertos.

Recogemos lo abordado en dicho artículo que esperemos sea de vuestro interés, sobre todo para aquellos que hacen Anestesia Pediátrica.

Generalidades sobre el empleo de Anestésicos Locales en niños

Para entender el efecto de los anestésicos locales en la población pediátrica primero debemos tener claro que dichos anestésicos locales son metabolizados principalmente por el citocromo P450 (CYP). Su isoforma principal: CYP3A4, se encarga de la Lidocaína y la Bupivacaina; siendo el CYP1A2 el encargado de metabolizar la Ropivacaína.
El CYP3A4 no es maduro al nacimiento. Por lo que, al mes de edad, la depuración intrínseca de la Bupivacaina es solo de $1 / 3$ del adulto y $2 / 3$ a los 6 meses. Por otro lado, el CYP1A2 no es completamente maduro hasta la edad de 3 años; por ello, la depuración de Ropivacaína no alcanza su máximo hasta la edad de los 8 años.

Recordad que el volumen de distribución de $\mathrm{AL}$ en neonatos e infantes es mucho mayor que en adultos; por ello, el riesgo de alcanzar una alta concentración del fármaco en el suero es raro tras la inyección única; sin embargo, el riesgo de acumulación después de una infusión continua o tras varias inyecciones está incrementado.

La fracción libre del AL será mayor en neonatos e infantes ya que presentan menos concentración de proteínas de transporte que los adultos.

El gasto cardiaco en los niños es mayor por lo que encontraremos una absorción farmacológica acelerada, al igual que se producirá una concentración inicial en plasma mayor. Por otro lado, reducirán la duración de acción. La dosis de AL en niños menores de 2 años deberá reducirse dado que presentan una frecuencia cardiaca mayor, con lo que incrementan la vulnerabilidad a la toxicidad cardiaca.

La duración del bloqueo espinal es menor en infantes que en adultos, ya que presentan un mayor volumen de líquido cefalorraquídeo; de hecho, se ha comprobado una relación entre la duración del bloqueo motor y la edad.

Respecto a los AL de larga duración debemos saber que la Ropivacaína, al igual que la Levobupivacaina, presenta menos cardiotoxicidad que la Bupivacaina, sin grandes diferencias en cuanto a la intensidad y duración de la analgesia. Por otro lado, la Ropivacaína 
y la Levobupivacaina producen un bloqueo motor menos intenso que la Bupivacaina.

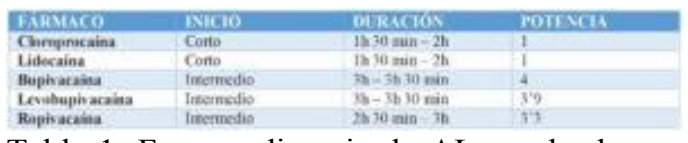

Tabla 1. Farmacodinamia de AL empleados en Anestesia Locorregional.

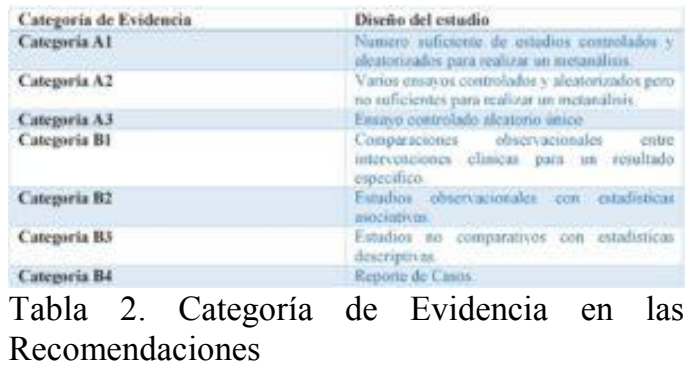

\section{Técnicas de Anestesia Locorregional}

\section{1.) Bloqueo Neuroaxial y Anestésicos Locales}

\section{A) Anestesia Espinal:}

Cuando hablamos de Anestesia Espinal, refieren que lo más empleado (Evidencia B3) es la Tetracaína 0'5\% en dextrosa al 5\%: pautándolo a 0'13 $\mathrm{ml} / \mathrm{Kg}<4 \mathrm{Kg}$ y a 0’07 ml/Kg $>4 \mathrm{Kg}$.

Por otro lado, no presentan inconveniente en el empleo de Bupivacaina hiperbárica o isobárica, Levobupivacaina isobárica o Ropivacaína, aunque ya sabemos que la Bupivacaina es la más cardiotóxica, por lo que la dejaríamos para la última opción si disponemos de las otras. La Ropivacaína recomiendan emplearla (Evidencia B3) a dosis: 0'5 mg/Kg. La Bupivacaina 0'5\% su recomendación es (Evidencia B3):

$$
\begin{array}{lrcrr}
1 & \mathrm{mg} / \mathrm{Kg} & < & 5 & \mathrm{Kg} \\
0{ }^{\prime} 4 & \mathrm{mg} / \mathrm{Kg} & 5 \mathrm{Kg} & - & 15 \mathrm{Kg} \\
0{ }^{\prime} 3 & \mathrm{mg} / \mathrm{Kg}>15 \mathrm{Kg} \\
\\
\text { B) }
\end{array}
$$

Presentan, que de los datos recogidos, el $25 \%$ de los pacientes en los que se emplea un bloqueo caudal reciben una dosis de AL que puede provocarles toxicidad. Por ello plantean que el volumen administrado sea modificado para alcanzar el nivel de dermatomo deseado de acuerdo con la fórmula de Armitage, evitando el empleo de concentraciones elevadas, priorizando el volumen en su lugar:

$-0,5 \mathrm{ml} / \mathrm{Kg}$ para alcanzar dermatomos Sacros

-1 $\mathrm{ml} / \mathrm{Kg}$ para alcanzar dermatomos Lumbares

$-1 ' 25 \mathrm{ml} / \mathrm{Kg}$ para alcanzar dermatomos Torácicos bajos

La Ropivacaína 0 '2\% (2mg/ml) o Levobupivacaina/Bupivacaina $\quad 0{ }^{\prime} 25 \%$ (2'5 mg/ml) son recomendados para la realización de bloqueos caudales en niños y no deben exceder $2 \mathrm{mg} / \mathrm{Kg}$ de Ropivacaína o 2'5 mg/Kg Bupivacaina o Levobupivacaina (Evidencia B2)

\section{C) Epidural Lumbar o Torácica:}

Al igual que la epidural caudal, el empleo de Ropivacaína 0’2\% o Levobupivacaina / Bupivacaina 0'25\% es igual en la epidural lumbar o torácica en niños. Se suele emplear una dosis de 0'5 mL/Kg y 0'3 mL/Kg como bolo inicial en la epidural Lumbar y Torácica respetivamente. $\mathrm{Y} \quad 0^{\prime} 25 \mathrm{ml} / \mathrm{Kg}$ como rescate para conseguir analgesia intraoperatoria.

Las propiedades de amortiguación del espacio epidural son importantes en tanto que evitan el aumento rápido de concentración. La dosis máxima que no se debe sobrepasar para epidural torácica y lumbar en niños es de 1'7 $\mathrm{mg} / \mathrm{Kg}$ Ropivacaína, Bupivacaina o Levobupivacaina (Evidencia B3), valores muy inferiores a la epidural lumbar que comentábamos previamente. 
D) Infusión Continua en Anestesia Epidural

La infusión epidural con Ropivacaína provee alivio del dolor satisfactorio en neonatos y niños menores de 1 año. La concentración de Ropivacaína libre no se influencia por la duración de la infusión, por lo que su empleo es seguro en el postoperatorio en infusiones de 48 a 72 horas. Los niveles de Ropivacaína libre son mayores en neonatos que en bebes, pero muy por debajo de las concentraciones del umbral para la toxicidad del sistema nervioso central en adultos, es decir, mayor o igual a 0’35 mg/L.

En la primera semana de vida la infusión de Ropivacaína debe usarse con más precaución. Debido a las preocupaciones existentes sobre la toxicidad debida a la acumulación de $\mathrm{AL}$ amidas en bebes y niños pequeños; la Cloroprocaina podría ser una alternativa.

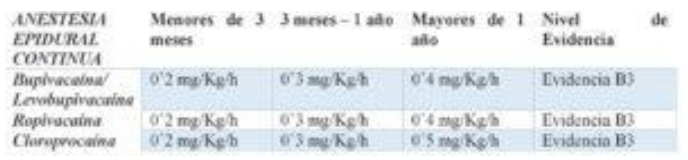

Tabla 3. Recomendaciones sobre dosis de AL en Anestesia Epidural Continua en la población pediátrica

\section{2.) Inyección Única de $A L$ para Nervios Periféricos y Bloqueo de plano fascial}

No es mucha la información que existe en publicaciones sobre la dosificación de anestésicos locales en la población pediátrica cuando hablamos de bloqueos periféricos.

Respecto al intercostal sabemos que se trata del bloqueo con el mayor ratio de reabsorción, y por tanto, con un elevado potencial de toxicidad sistémico. Por otro lado, las dosis más altas de AL (2'5 $\mathrm{mg} / \mathrm{Kg}$ ) empleadas en bloqueos nerviosos intercostales alcanzan niveles plasmáticos por debajo de los niveles potencialmente tóxicos.

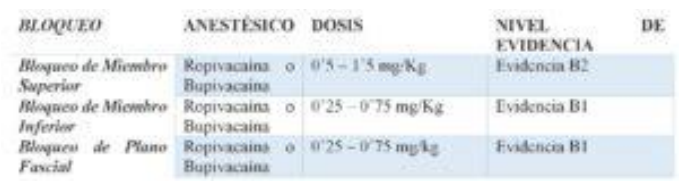

Infusión continua de AL en Nervios Periféricos y Bloqueos de plano fascial

La infusión continua de AL para nervio periférico o bloqueos de plano fascial pueden realizarse con éxito y seguridad con 0 '2\% Ropivacaína o Bupivacaina empleando una dosis de infusión de 0'1 - 0’3 mg/Kg por hora (Evidencia B3)

\section{Bloqueo paravertebral ¿Qué sabemos hasta ahora en la población pediátrica?}

Aunque no se habla en la guía de bloqueos específicos, se comentan varias cosas en ella respecto al bloqueo Paravertebral que considero importante remarcar:

- Los estudios han demostrado que el bloqueo paravertebral torácico (como una inyección única o infusión continua) se puede utilizar con éxito en niños, incluidos los bebés.

- Por otro lado, la evaluación de la farmacocinética de la lidocaína y la bupivacaina después de una sola inyección $\mathrm{o}$ infusión paravertebral continua en niños ha demostrado que los niveles plasmáticos totales de bupivacaina potencialmente altos eran frecuentes en lactantes con infusiones de bupivacaina al $0,25 \%$ a $0,25-0,5$ $\mathrm{mg} / \mathrm{kg}$ por hora.

- Un estudio de seguimiento mostró que la analgesia post-toracotomía efectiva se logró con una concentración más baja (bupivacaina $0.125 \%$ con epinefrina 1 : $400,000)$ y dosificación $(0.2 \mathrm{~mL} / \mathrm{kg}$ por hora durante 48 horas), pero la 
acumulación sistémica seguía teniendo lugar, y concentraciones mayores de 3 $\mu \mathrm{g} / \mathrm{mL}$ se registraron en algunos bebés a las 48 horas.

Por ello, vemos que es una técnica que a pesar de poder emplearse en la población pediátrica aún debe seguir estudiándose la concentración del AL y su dosificación para evitar acumulo de dicho fármaco. Antes de realizarla, actualmente, se debe valorar riesgobeneficio de la técnica en esta población.

\section{Adyuvantes para bloqueos neuroaxiales y periféricos}

Los anestésicos locales de acción prolongada (Bupivacaina,

Levobupivacaina y Ropivacaína) tienen una duración de acción limitada: 4-12 horas, frente a un dolor postoperatorio que puede alcanzar las 48-72 horas, por ello, para prolongar el efecto del bloqueo podemos usar técnicas de catéter, mediante bolos o infusión continua de AL. Pero en pediatría no siempre es necesaria esta técnica, por lo que podemos aumentar el bloqueo empleando adyuvantes.

El empleo de adyuvantes ofrece numerosas ventajas como: prolongar el efecto del bloqueo y el analgésico, reducir las necesidades de anestesia general, mejorar la confortabilidad postoperatoria...:

- La Clonidina (1-2ug/Kg) y la morfina $(10-30 \mathrm{ug} / \mathrm{Kg})$ pueden emplearse intratecalmente para prolongar la duración del bloqueo espinal en niños. La dosis debe ser la mínimamente necesaria para lograr beneficios minimizando en lo posible los efectos adversos (Evidencia A2)

- Se ha empleado Ketamina racémica (0'5 mg/Kg) y S-Ketamina como complemento neuroaxial en niños. Sin embargo, la Ketamina no se recomienda para uso intratecal en neonatos y lactantes debido a la posibilidad de una mayor apoptosis neuronal en médula espinal. Dado que los datos de toxicidad son limitados en los niños, la dosis empleada debe ser la mínima necesaria para lograr beneficios con los menores efectos adversos (Evidencia B3).

- La Dexmedetomidina se ha empleado para prolongar la analgesia postoperatoria como complemente de los bloqueos neuroaxiales (ej: bloqueo caudal). Dado que los datos sobre toxicidad son muy limitados en los niños, la dosis empleada debe ser la mínima necesaria para lograr beneficios minimizando los efectos adversos (Evidencia A2).

- Aunque el empleo de opiáceos sintéticos (Ej: Fentanilo y Sulfentanilo) son comunes como adyuvantes en el contexto de la analgesia epidural en adultos, no existe evidencia de que los opiáceos sintéticos produzcan algún efecto relevante empleados como adyuvantes de los bloqueos caudales en los niños. El fentanilo no potencia el efecto de la Bupivacaina o la Ropivacaína en el contexto del bloqueo caudal (Evidencia A2).

- Los corticoesteroides como la Dexametasona se han empleado en pequeños estudios en pacientes pediátricos como adyuvante neuroaxial. Con base en la evidencia clínica actual, el comité asesor no recomienda el uso de corticosteroides como adyuvante neuroaxial en niños (Evidencia B2).

\section{Conclusión}

A medida que se vaya disponiendo de nuevos datos, posiblemente se irán necesitando versiones más actualizadas de la guía. Lo que se ha intentado con esta guía es cubrir las técnicas de 
anestesia regional más comunes en niños, por ello no están cubiertos todos los escenarios posibles; no proporcionan información detallada o prescriptiva para bloqueos específicos.

\section{Bibliografía}

Suresh S, Ecoffey C, Bosenberg A, Lonnqvist PA, de Oliveira GS Jr, de Leon Casasola O, et al. «The European Society of Regional Anaesthesia and Pain Therapy/American Society of Regional Anesthesia and Pain Medicine Recommendations on Local Anesthetics and Adjuvants Dosage in Pediatric Regional Anesthesia». Regional Anesthesia and Pain Medicine 43, n.o 2 (febrero de 2018): 211 16.

https://doi.org/10.1097/AAP.000000000000070 2. (PubMed)

Correspondencia al autor

Marcos Salmerón Martín

Marcos.sm9290@gmail.com

MIR 3e Anestesia y Reanimación

H.C.U. Virgen de la Arrixaca, Murcia

Aceptado para blog en octubre de 2018. 